\title{
Retrospective Data Capture
}

National Cancer Institute

\section{Source}

National Cancer Institute. Retrospective Data Capture. NCI Thesaurus. Code C142673.

Data that is collected after the fact, originating from subject recall or memory. 